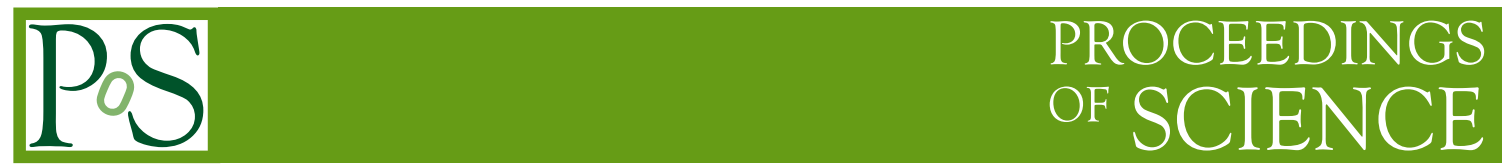

\title{
Performance of the CMS Level-1 Trigger during Run 2
}

\author{
Hyejin Kwon ${ }^{a, 1, *}$ \\ ${ }^{a}$ Seoul National University, Seoul, Korea \\ E-mail: hyejin.kwon@cern.ch
}

The CMS Level-1 (L1) Trigger system was upgraded in 2016 in order to cope with the threefold increase in peak luminosity of Run 2 compared to Run 1 . The upgraded trigger features advanced clustering, calibration, and particle identification techniques. More sophisticated algorithms, for example involving the invariant mass of pairs of L1 candidates, were also implemented. The upgrades reduced the trigger rate from background processes and increased the efficiency for a variety of physics signals. This poster highlights a number of improvements that the upgraded L1 trigger provided during the Run 2 program at CMS.

40th International Conference on High Energy physics - ICHEP2020

July 28 - August 6, 2020

Prague, Czech Republic (virtual meeting)

${ }^{1}$ On behalf of the CMS Collaboration

* Speaker 


\section{The CMS Level-1 trigger architecture}

The CMS experiment [1] implements a sophisticated two-level trigger system which reduces event rates by a factor of $10^{5}$. It consists of the Level-1 (L1) trigger and the High Level Trigger (HLT). The L1 trigger is a hardware trigger which collects the information from the calorimeter and muon chambers. The $\mathrm{L} 1$ trigger selects up to $100 \mathrm{kHz}$ of the most interesting events with a latency of $4 \mu \mathrm{s}$.

Figure 1 shows the CMS L1 trigger system during Run 2. The muon trigger system includes three muon track finders for each geometrical region. It reconstructs muons in the barrel (BMTF), overlap (OMTF), and endcap (EMTF) regions of the detector, and sends them to the global moun trigger for final muon selection. The calorimeter trigger consists of two layers. The Layer-1 receives and calibrates the local energy deposits which are sent by the electromagnetic calorimeter (ECAL) and hadron calorimeter (HCAL). The Layer-2 uses these trigger primitives (TPs) to reconstruct and calibrate the physics objects such as electrons, tau leptons, jets. Lastly, the global trigger combines the information from global muon trigger and calorimeter trigger.

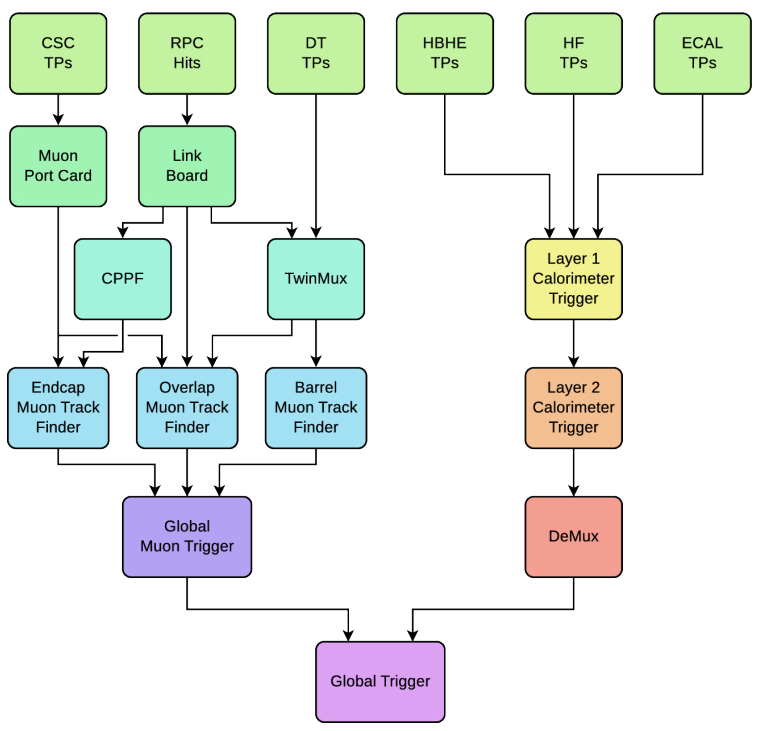

Figure 1: Diagram of the upgraded CMS Level-1 trigger system during Run 2.

\section{Performance of the Level-1 muon trigger}

The CMS muon system consists of three types of muon detectors: Drift Tube (DT) chambers, Resistive Plate Chambers (RPC) and Cathode Strip Chambers (CSC). Using distinct regions, the L1 muon trigger improved its resolution and redundancy. The BMTF in the barrel region uses a road search extrapolation track finder. The OMTF and the EMTF use a pattern based track finder and the EMTF also uses a boosted decision tree (BDT) based $p_{T}$ assignment. In Figure 2, a single muon trigger efficiency for the typical $\mathrm{L} 1$ threshold $22 \mathrm{GeV}$ is shown for each region. High efficiency on the plateau and sharp turn-on curves represent a good performance of the L1 muon trigger system. 


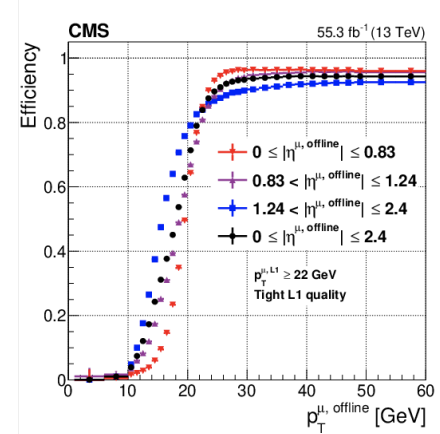

Figure 2: The L1 muon trigger efficiency for data as a function of the offline reconstructed muon $p_{T}$ for each region: barrel region in red, overlap region in purple, endcap region in blue, and the total in black.
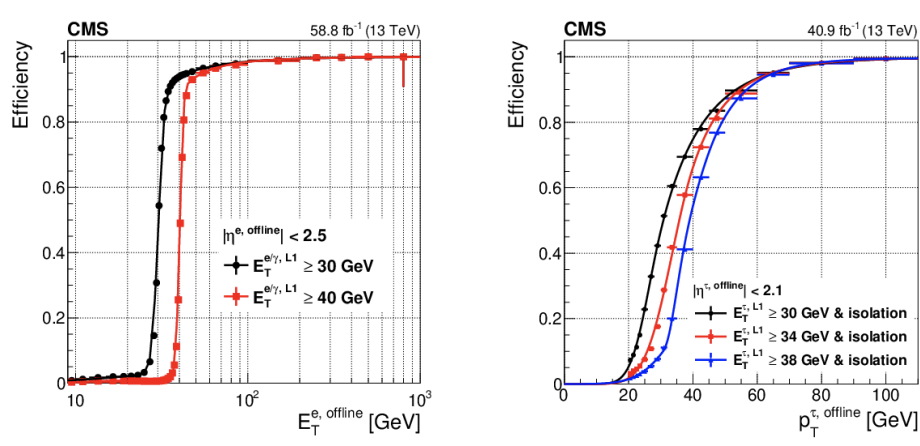

Figure 3: The L1 e/ $\gamma$ trigger effi- Figure 4: The L1 isolated $\tau$ trigciency as a function of the offline ger efficiency as a function of the reconstructed electron $E_{T}$ : an $E_{T}$ offline reconstructed $\tau p_{T}$ : a $p_{T}$ threshold of $30 \mathrm{GeV}$ in black, and threshold of $30 \mathrm{GeV}$ in black, 34 of $40 \mathrm{GeV}$ in red. $\mathrm{GeV}$ in red, and of $40 \mathrm{GeV}$ in blue.

\section{Performance of the Level-1 calorimeter trigger}

The e $/ \gamma$ trigger uses a dynamic clustering algorithm around the seed (local energy maximum). Calibration is done depending on $E_{T}, \eta$ and shape of the cluster. Figure 3 shows the e/ $\gamma$ trigger efficiency as a function of the offline reconstructed electron $E_{T}$ for thresholds of 30 and $40 \mathrm{GeV}$. It shows high plateau efficiency and its sharp turn-on reflects a good energy resolution. The $\tau$ trigger uses the e $/ \gamma$ clustering as a baseline. A $\tau$ candidate is considered as isolated if the $E_{T}$ in the isolation area is smaller than a chosen value. Figure 4 shows the isolated $\tau$ trigger efficiency for several thresholds. It also shows a good performance. The jet trigger clustering uses a 9x9 trigger tower sliding window centered on the jet seed. The pileup subtraction is applied to the jets.

\section{References}

[1] CMS Collaboration, "The CMS experiment at the CERN LHC", JINST 3 (2008) S08004, doi:10.1088/1748-0221/3/08/S08004.

[2] CMS Collaboration, "Performance of the CMS muon detector and muon reconstruction with proton-proton collisions at $\sqrt{s}=13$ TeV", JINST 13 (2018) P06015, doi:10.1088/17480221/13/06/P06015, arXiv:1804.04528.

[3] CMS Collaboration, "Performance of the CMS Level-1 trigger in proton-proton collisions at $\sqrt{s}=13 \mathrm{TeV}$ ", JINST 15 (2020) P10017, doi:10.1088/1748-0221/15/10/P10017, arXiv:2006.10165. 